\title{
Global mean estimation using a self-organizing dual-zoning method for preferential sampling
}

\author{
Yuchun Pan • Xuhong Ren • Bingbo Gao • Yu Liu • \\ YunBing Gao • Xingyao Hao $\cdot$ Ziyue Chen
}

Received: 7 October 2014 / Accepted: 9 February 2015 /Published online: 19 February 2015

(C) Springer International Publishing Switzerland 2015

\begin{abstract}
Giving an appropriate weight to each sampling point is essential to global mean estimation. The objective of this paper was to develop a global mean estimation method with preferential samples. The procedure for this estimation method was to first zone the study area based on self-organizing dual-zoning method and then to estimate the mean according to stratified sampling method. In this method, spreading of points in both feature and geographical space is considered. The method is tested in a case study on the metal $\mathrm{Mn}$
\end{abstract}

Y. Pan • B. Gao $(\bowtie) \cdot$ Y. Liu $\cdot$ Y. Gao $\cdot$ X. Hao

Beijing Research Center for Information Technology in

Agriculture, Beijing Academy of Agriculture and Forestry

Sciences, Shuguang Huayuan Middle Road 11\#,

Beijing 100097, China

e-mail: gaobb@nercita.org.cn

Y. Pan $\cdot$ B. Gao $\cdot$ Y. Liu $\cdot$ Y. Gao $\cdot$ X. Hao

National Engineering Research Center for Information

Technology in Agriculture, Shuguang Huayuan Middle Road

11\#, Beijing 100097, China

X. Ren

Department of Computer Science and Engineering, North China Institute of Aerospace Engineering, Aimin East Road 133\#, Langfang City, Hebei Province 065000, China

B. Gao

Institute of Geographic Sciences \& Nature Resources

Research, Chinese Academy of Sciences, A11 Datun Road,

Chaoyang Beijing 100101, China

Z. Chen

College of Global Change and Earth System Science, Beijing

Normal University, 19 Xinjiekouwai Street, Haidian

Beijing 100101, China concentrations in Jilin provinces of China. Six sample patterns are selected to estimate the global mean and compared with the global mean calculated by direct arithmetic mean method, polygon method, and cell method. The results show that the proposed method produces more accurate and stable mean estimates under different feature deviation index (FDI) values and sample sizes. The relative errors of the global mean calculated by the proposed method are from 0.14 to $1.47 \%$ and they are the largest (4.83-8.84\%) by direct arithmetic mean method. At the same time, the mean results calculated by the other three methods are sensitive to the FDI values and sample sizes.

Keywords Preferential sampling · Global mean estimation · Self-organizing dual-zoning method

\section{Introduction}

The estimation of population parameters based on sampling data is an important task in the investigation and monitoring of natural resources and the environment. The sample mean from a group of observations that are assumed to be non-preferential is an estimation of the population mean (Wang et al., 2009, 2010; Li et al., 2008). In practice, however, samples occasionally represent preferential measurements due to economic and geographic limitations (Merckx et al., 2011; Kamiran and Calders, 2010; Botta-Dukát et al. 2007; Michalcová et al. 2011; Menezes, 2009). For preferential samples, the estimation would be biased if applying an arithmetic 
average (Isaaks and Srivastava 1989; Diggle et al., 2010). Therefore, research on reasonable methods for computing the global mean estimation under preferential sampling conditions is necessary.

The ratio estimator is a statistical parameter that is often used to estimate the mean based on sampling theory. This method is very simple, and it has been shown that the ratio estimator is more efficient under well-known circumstances than the conventional method of estimation using the sample mean (Rao, 1981). Subsequently, unbiased ratio estimators that consider linear combinations and other approaches have been proposed. Often, it is advantageous to make use of prior knowledge at the estimation stage (Shabbir and Yaab, 2003; Gupta and Shabbir, 2007). Therefore, when auxiliary variables are readily available and their population distributions are easily obtained, the use of this estimator is practical and feasible.

The assignment of an appropriate weight to each sampling point is essential to global mean estimation. Thus, for conditions where auxiliary variables are not available, zoning methods have been proposed to solve this problem. To overcome the deficiencies of the arithmetic mean method, researchers have successively put forward two methods that consider only the geographical space distribution of the sample, including the cell method (Deutsch, 1989) and polygonal method (Thiessen 1911). The contribution of each sample to the mean is directly proportional to the assigned spatial extent. Therefore, the estimation performance is improved to some extent (Journel, 1983; Dubois and Saisana 2002). However, Olea (2007) indicated that preferential samples are biased not only with respect to spatial location but also attribute values. The main cause of the mean deviation for preferential samples is the bias in feature space. When the representation of the attribute in feature space is ignored, sample weights on the global mean are not comprehensive.

In this paper, methods for global mean calculation are assessed with respect to the representativeness in both geographical space and feature attributions. Lin et al. (2005) proposed a dual clustering method that takes into account the attribute values and geographical space while considering that the clusters are continuous in spatial domain and similar in feature space. The similarity measurement accounts for geospatial attributes and non-geometric attributes such that the samples in a cluster have the same contribution to the global mean. In general, dual clustering has become a primary concern among many researchers (Tai et al., 2007). It is known that the self-organization feature map (SOFM) can achieve efficient clustering results (Kohonen 1990). Subsequently, Jiao et al. (2011) adopted the SOFM method, incorporated dual clustering, and improved this method to generate a more precise and meaningful result. Therefore, the overall goal of this study was to develop a dual zoning approach for obtaining an accurate global mean from preferential sample.

The rest of this paper is organized as follows: section 2 presents the data and proposes global mean estimation method under preferential sampling. The results and discussion are presented in section 3 , and section 4 draws the conclusions.

\section{Data and methodology}

Sites and data

The study area locates in three counties of the Siping (Lishu County, Gongzhulin County, and Tiedong District) in Jilin provinces of China. This area is an important transport hub in northeast China. The oreforming conditions are favorable, and the ore-bearing potential is high in the eastern and southern districts but low in other areas. The stratified heterogeneity of this region makes it a good experimental study area for preferential sampling. To produce preferential samples, minerals, roads, and industrial land use type with high metal concentration were sampled denser than other area. A sample with 305 points was collected and the Mn concentrations were determined by atomic fluorescence spectrometry. It is treated as the initial sample. From Fig. 1, it is readily apparent that the points are clustered in geographic space. We used six sets of samples for this experiment: besides the initial sample with 305 points, the other five samples were created by subsampling from the 305 points, and the sizes ranged from 24 to $47 \%$ of 305 . Each sample was drawn by first dividing the study area into subregions and then sampling unequally in each subregion. Maps of the samples are shown in Fig. 1.

Because the exhaustive data of Mn concentrations in the study area is not available, the sequential indicator simulation was used to generate a simulated dataset called reference dataset in this paper based on the initial sample to play as population. The sequential indicator simulation method can provide optimal interpolation 


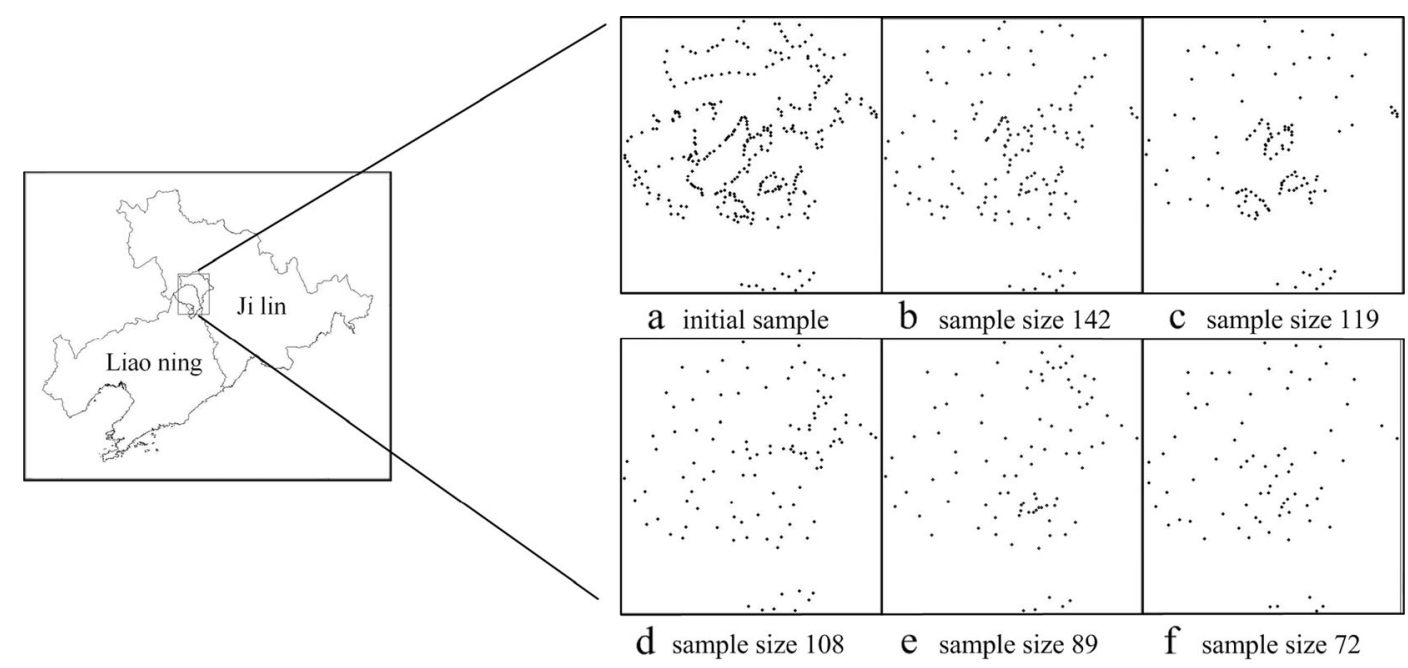

Fig. 1 a-f The location of the preferential samples

whereby measured data values are honored at their locations and local details of the spatial variation are not smoothed out. And unlike the sequential Gaussian simulation, it could produce better continuity for extreme values, both small values and high value by using different indicator variograms (Goovaerts, 1997). As shown in the thematic map in Fig. 2a, the clusters of high values and low values exist. Thus, the sequential indicator simulation can produce relative heterogeneous simulated dataset. With the Mn concentrations of 305 points of the initial sample as hard data and the experimental indicator variograms gotten, a $1000 \times 1000$ points dataset was generated using the sequential indicator simulation tool of WinGslib v.1.5. The reference dataset simulated is presented in Fig. 2b, and the spatially stratified heterogeneity is obvious. The reference dataset was taken as the population and distribution and global true value of Mn concentrations are drawn from it to evaluate the performance of different method.

\section{Hybrid distance and SOFM clustering}

An SOFM is composed of input and output layers and is used to organize clusters using competitive learning. The neuron of each output layer bears a set of connection weights which is a vector constituted with weights of attributes of input point on this neuron. The connection weights are used to choose the best matching layer for input points and are adjusted gradually during learning. By considering both spatial distances and attribute distances in SOFM, points with similar characteristics are classified into a cluster, and at the same time, they are adjacent in the geographic space.

Spatial distance and feature similarity are defined to delineate the neighboring relationships in the geographic domain and feature space. In this manner, the hybrid distance, i.e., a weighted distance for spatial distances and attribute distances, is defined. A dual spatial point is a point object in a geographic space with attribute values, which can be defined as:

$X_{N}=\left\{g_{N}^{1}, g_{N}^{2}, \ldots, g_{N}^{L}, a_{N}^{1}, a_{N}^{2}, \ldots, a_{N}^{T}\right\}$.

Where $\left\{\mathrm{g}_{N}^{1}, g_{N}^{2}, \ldots, g_{N}^{L}\right\}$ is the geographic space ( $L$ is number of spatial dimensions, usually set to $1,2$, or 3$),\left\{a_{N}^{1}, a_{N}^{2}, \ldots, a_{N}^{T}\right\}$ is the feature space, and $T$ is the number of attributes. The attribute considered in case study of this paper is only the target variable, that is the Mn concentration. In application, ancillary data can also be used to determine the zones.

The geospatial distance between two points is defined as the Euclidean distance, $D_{i j}^{(S)}$, and the feature distance between two points is given by:

$D_{i j}^{(A)}=\sqrt{\sum_{t=1}^{T} \omega_{d}\left(a_{i}^{t}-a_{j}^{t}\right)^{2}}$.

where $\omega_{t}$ is the weight of attribute $t, \sum_{t=1}^{T} \omega_{t}=1$, and $a_{i}^{t}$ and $a_{j}^{t}$ are the attributes of the $i$ th and $j$ th points, 


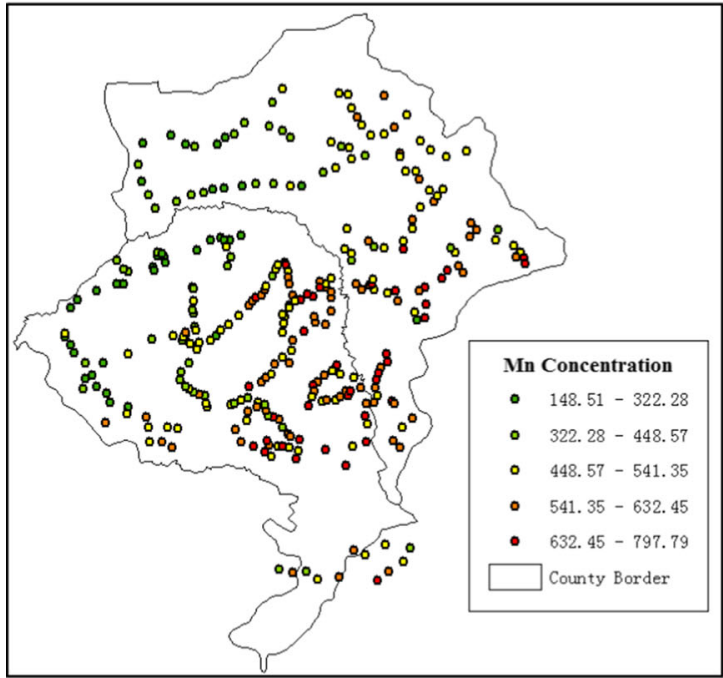

(a)

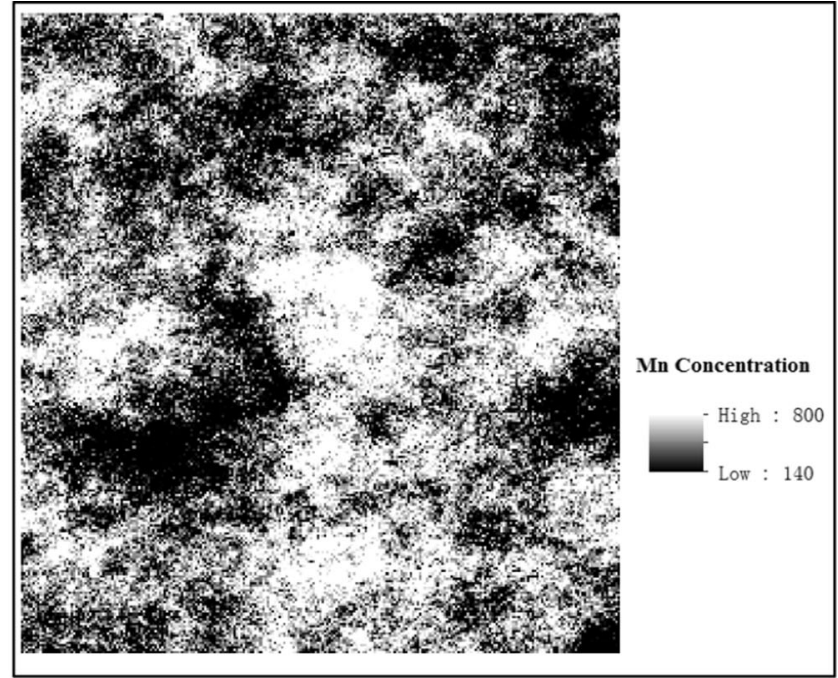

(b)

Fig. 2 The hard data and simulated reference dataset a map of hard data and $\mathbf{b}$ reference dataset created by sequential indicator simulation

respectively. The hybrid distance, $D_{i j}$, between the $i$ th and $j$ th points is given as:

$D_{i j}=w_{s} D_{i j}^{(S)}+w_{a} D_{i j}^{(A)}$.

Where $w_{a}$ and $w_{s}$ represent the weights of feature and geographic space, respectively, and $w_{S}+w_{a}=1$.

Global mean estimation method using a self-organizing dual-zoning method

In this study, the global mean estimation using a selforganizing dual-zoning method for preferential sampling consists of two steps: zoning by self-organizing dual-zoning method and the subsequent estimation of the global mean. The details of each step are introduced in following parts.

\section{Self-organizing dual-zoning method}

Before zoning, the number of zones to be divided should be determined. The following formula can be used to calculate the number:

$N=\operatorname{Int}\left(D_{\max }^{(A)} / A_{0}\right)+1$.

Where $N$ is the zoning number, $D_{\max }^{(A)}$ is the maximum distance in feature space, Int is a function for retrieving the integer portion of a number, and $A_{0}$ is the acceptable clustering dispersion in the feature space.
The self-organizing dual-zoning method can be realized in following four steps:

1. Clustering using SOFM

(a) Choose the weights and construct the hybrid distance by combining the geometric distance and attribute value similarity.

(b) Standardize the spatial coordinates and attribute values and initialize connection weights with random numbers between 0 and 1 .

(c) Randomly select a point as the input, and use the hybrid distance to determine the best matching point.

(d) Adjust the connection weights using the neighborhood and learning rate functions; then go to (c) until the network converges.

(e) Classify all sampling points using the converged network in learning stage.

2. Generate a Voronoi diagram of the sampling point and determine the adjacent geospatial relationships based on the edge-sharing between the polygons.

3. Aggregate the polygons that belong to the same cluster and perform preliminary zoning.

4. Detect the scattered and small polygons and amalgamate them into the nearest zone based on the maximum sharing boundary rule.

These steps are required to obtain the zone result after dual clustering: Voronoi diagram construction, polygon aggregation, and amalgamation. Polygon aggregation 
combines polygons within a specified distance to each other into new polygons. This operation is used to merge the Voronoi polygons belonging to the same cluster. The amalgamation operation computes the boundary of the union of sets of polygons. Outliers are merged into the adjacent cluster. The confirmation of adjacent polygons in the Voronoi diagram of the point set is based on the boundary or point sharing rule. We then merge the adjacent polygons in the same class. Finally, outliers are amalgamated into the other adjacent zone according to the maximum sharing boundary rule.

\section{Global mean estimation}

Based on the zoning result of self-organizing dual-zoning method, the global mean is estimated according to the stratified sampling method. The mean is calculated in the same zone using the arithmetic mean method, and the global mean is obtained according to Eq. (5), in which the weight of each zone is the ratios of zone area to the overall area.

$M_{\mathrm{glo}}=\left(\sum_{i=1}^{M} a_{i} \frac{1}{N_{i}} \sum_{j=1}^{N_{i}} d_{j}\right) / \sum_{i=1}^{N_{i}} a_{i}$.

Where $M$ is the number of clusters, $N_{i}$ is the number of sampling points in the $i$ th cluster, $a_{i}$ is the area of the $i$ th cluster, and $d_{j}$ is the attribute of $j$ th point in cluster $N_{i}$. The preferential properties in feature space and geographic space are fully considered in this method.

\section{Validation of calculation}

\section{The feature deviation index}

Probability-probability (PP) plot and quantilequantile (QQ) plots can reveal the relationships of the feature distribution between a sample and population. To quantify the feature similarity of a sample and population, the feature deviation index (FDI) is defined as the standard residual obtained from the fitting of the scatter plot of QQ using the line $y=x$. The FDI is calculated as:

$$
\mathrm{FDI}=\left[\sum_{i=1}^{N}\left(v_{\mathrm{sam}}(i)-v_{\mathrm{pop}}(i)\right)^{2}\right]^{1 / 2} /(N-1) \text {. }
$$

Where $v_{\text {sam }}$ is the values (quartiles) of the sample, $v_{\text {pop }}$ is the corresponding quartiles of the population, and $N$ is the sample size.

Taking the reference dataset as the population, we computed the FDI values for the six preferential samples. These results are shown in Table 1.

\section{The relative error of the global mean}

The accuracy of the obtained global mean was evaluated by the relative error of the global mean which is defined as:

$E_{\text {gm }}=\left|\left(M_{\text {real }}-M_{\text {est }}\right) / M_{\text {real }}\right| \times 100 \%$.

Where $M_{\text {real }}$ is the real mean of the target population, and $M_{\text {est }}$ is the global mean estimated for the samples.

\section{The cell method and polygonal method}

The purpose of the simulation experiment was to study the effectiveness of our method; thus, we employed the cell and polygonal methods. For the cell method, the cell sizes ranging from 0 to $75 \mathrm{~km}^{2}$ were used because the space of all data is greater than $0 \mathrm{~km}$ and the largest size corresponds to half of the research scope. The polygonal method was applied using the software ArcGIS v. 9.3.

\section{Results and discussion}

Analysis of samples

The six samples shown in Fig. 1 are intuitively clustered in the spatial domain. The statistical characteristics of the reference dataset and the generated six sampling datasets are shown in Table 1. The arithmetic means of the six sampling datasets are all much larger than that of the reference dataset. In addition, the range of the attribute values has narrowed to a certain extent for the preferential samples. The variation in the standard deviations is large, which is affected by the mean values. Figure 3 shows the cumulative probability curves. The plotted values for the reference dataset mainly lie along a straight line, whereas the cumulative probability curves of preferential sampling datasets show a poor linear fit. The cumulative probability values of the six sampling datasets are all less than that of the reference dataset at values less than $500 \mathrm{mg} / \mathrm{kg}$ of $\mathrm{Mn}$, and the $\mathrm{Mn}$ 
Table 1 The original statistical characteristic values and the global mean of Mn concentrations estimated by three methods

\begin{tabular}{|c|c|c|c|c|c|c|c|c|c|}
\hline Sample size & Min & Max & Mean & Median & Std. dev & FDI & Cell_mean & Polygonal_mean & Zone_mean \\
\hline $1,000,000$ & 140.00 & 800.00 & 488.50 & 509.75 & 200.91 & - & - & - & - \\
\hline 305 & 148.51 & 797.79 & 494.50 & 505.55 & 131.03 & 77.93 & 480.18 & 493.21 & 487.84 \\
\hline 142 & 165.87 & 775.65 & 520.04 & 539.08 & 123.23 & 86.17 & 489.5 & 502.33 & 494.00 \\
\hline 119 & 220.62 & 797.79 & 531.62 & 537.80 & 117.81 & 100.86 & 480.63 & 497.73 & 486.62 \\
\hline 108 & 148.51 & 797.79 & 512.05 & 534.33 & 131.61 & 82.98 & 482.54 & 496.14 & 491.69 \\
\hline 89 & 219.50 & 797.79 & 532.67 & 538.82 & 127.90 & 94.48 & 495.02 & 502.87 & 495.68 \\
\hline 72 & 165.87 & 752.03 & 520.51 & 552.43 & 139.53 & 90.93 & 507.68 & 514.47 & 494.89 \\
\hline
\end{tabular}

The Min, Max, Mean, Median, and Std. dev are descriptive statistics of corresponding data; FDI is the feature deviation index of samples; Cell_mean, Polygonal_mean, and Zone_mean are the global mean estimate of cell method, polygonal method, and our method

concentrations gradually converge when the probability is greater than $500 \mathrm{mg} / \mathrm{kg} \mathrm{Mn}$. Then the value reaches $600 \mathrm{mg} / \mathrm{kg} \mathrm{Mn}$, the probabilities of the sampling datasets are all greater than that of the reference dataset. Figure 3 shows that the sampling datasets all have deviations in feature space compared with the reference dataset. Thus, it is concluded that the six sampling datasets are preferential in feature space and geographic space, which is in agreement with the requirement of the experiment.

The zoning results of dual-zoning method

Figure 4 shows the results of the self-organizing dualzoning method. Different clusters are delineated by different shapes. The Voronoi polygons are aggregated according to the clustering results. After detecting the outliers, the fragmentary polygons were merged into those having the longest shared boundary. The revised zoning boundaries of the clusters were then produced

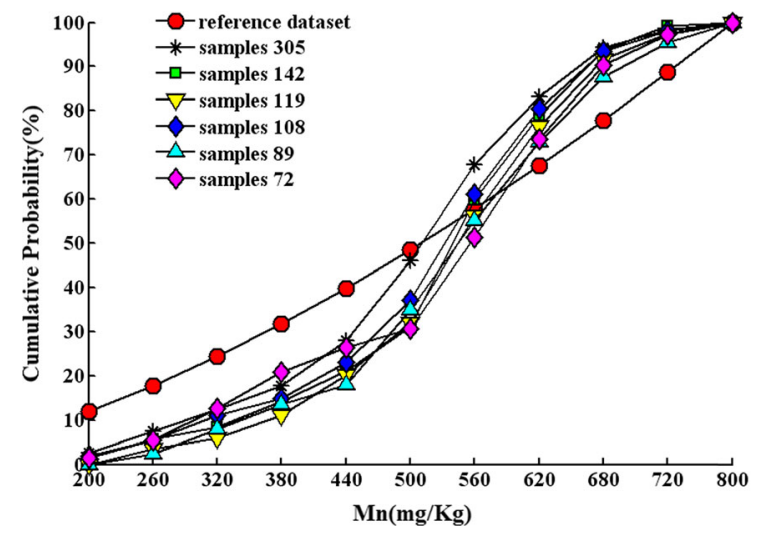

Fig. 3 Cumulative distribution of reference dataset and samples
(Fig. 4b). The global mean results estimated using the self-organizing dual-zoning method are displayed in Table 1.

The relative error of the global mean estimation

The global mean results calculated by these methods were shown in Table 1 . We used the mean of the reference dataset as the real mean to calculate the relative error of the global mean estimations. We evaluated the global mean estimation methods for preferential samples from two perspectives: the sample size and feature deviation.

\section{The effect of the FDI on the mean estimation}

Compared to the arithmetic mean method, the precision of the mean calculated by the other three methods was greatly improved. The relative errors of the global mean derived for each preferential dataset were shown in Fig. 5. The $x$-axis in Fig. 5a show the FDI values associated with each dataset. The results of the arithmetic mean method were sensitive to the FDI values. It was readily apparent that the errors increased substantially as the FDI values increased. The results of cell method and polygonal method generally varied in unison. In contrast, these two methods reduced the errors of the global mean to some extent. However, when the FDI value was 90.93, the error of the two methods were significantly increased. This outcome may be explained by the fact that the sample size was too small; i.e., when the sample size is below a certain level, the sample size effects are increased and become the main source of error. Overall, the stability of these two methods is relatively poor, 


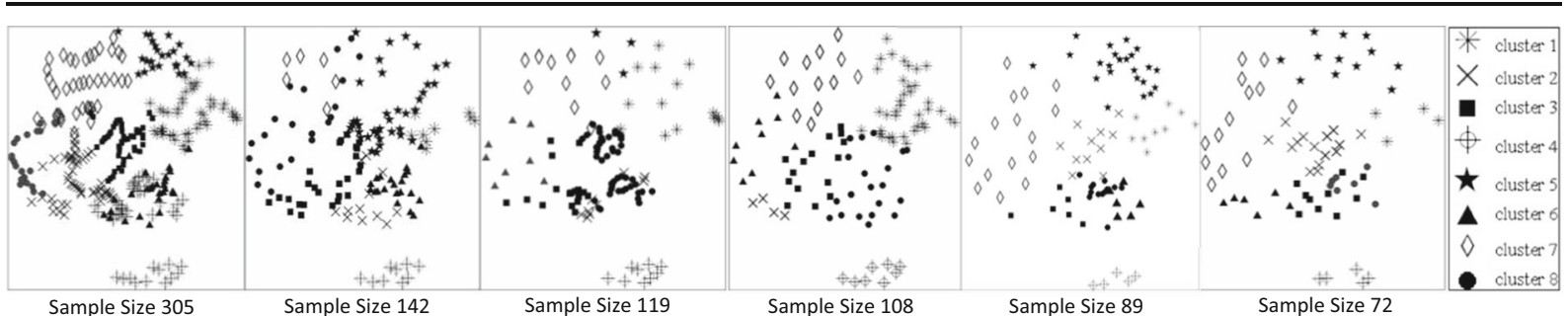

(a)

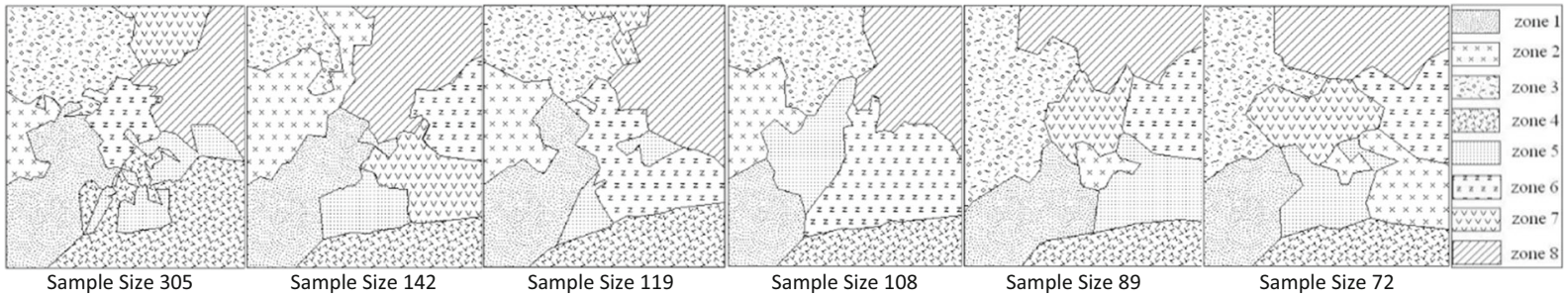

(b)

Fig. 4 The results of the self-organizing dual-zoning method. a Clustering results generated by the SOFM. b Final zoning results

especially for small sample sizes. For the self-organizing dual-zoning method, regardless of the FDI value, the
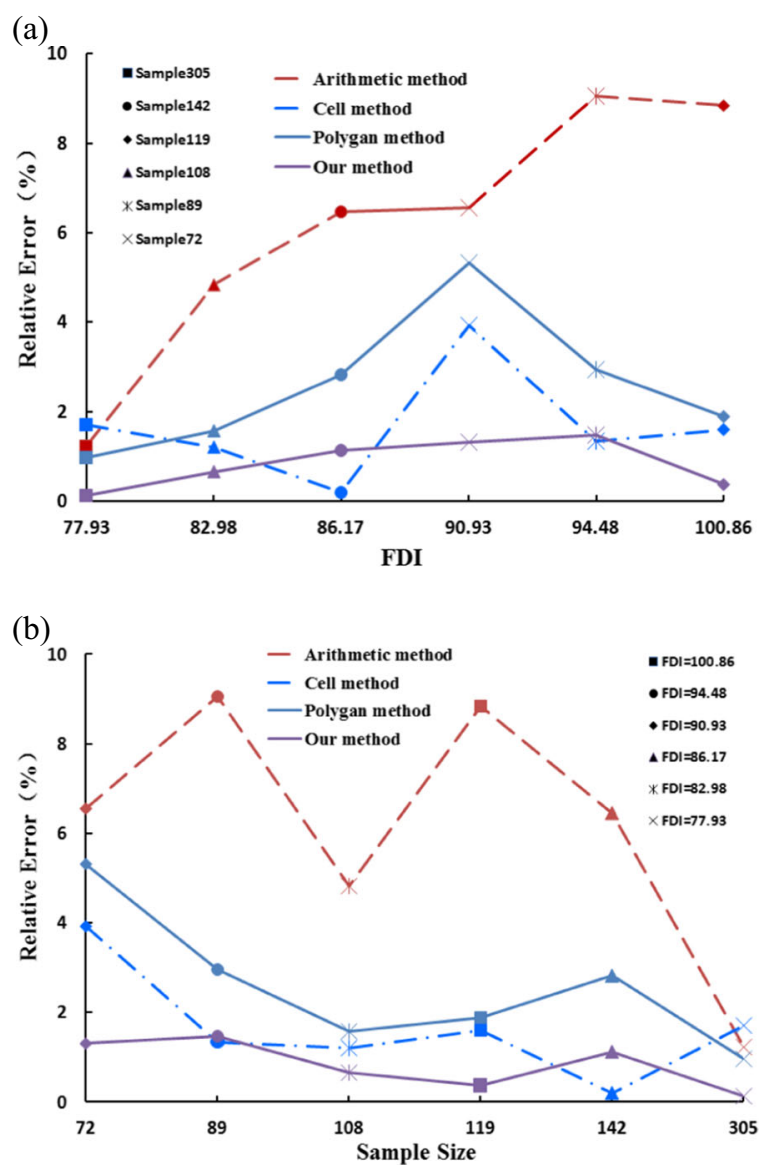

Fig. 5 The relative error of the mean for the four evaluated methods as a function of the $\mathbf{a}$ FDI and $\mathbf{b}$ sample size errors are small and stable. In conclusion, Fig. 5a demonstrates that the dual-zoning method can effectively reduce the influence of FDI on the global mean estimation and can be used to obtain more accurate mean results.

\section{The impact of the sample size on the mean estimation}

The $\mathrm{x}$-axis shows the sample size which was associated to each dataset in Fig. 5b. It is can be seen that errors of the mean result calculated by arithmetic mean method have a decreasing trend along with the increase of sample size. Nonetheless, the results also have fluctuation since the impact of FDI value which is given rise to the non-

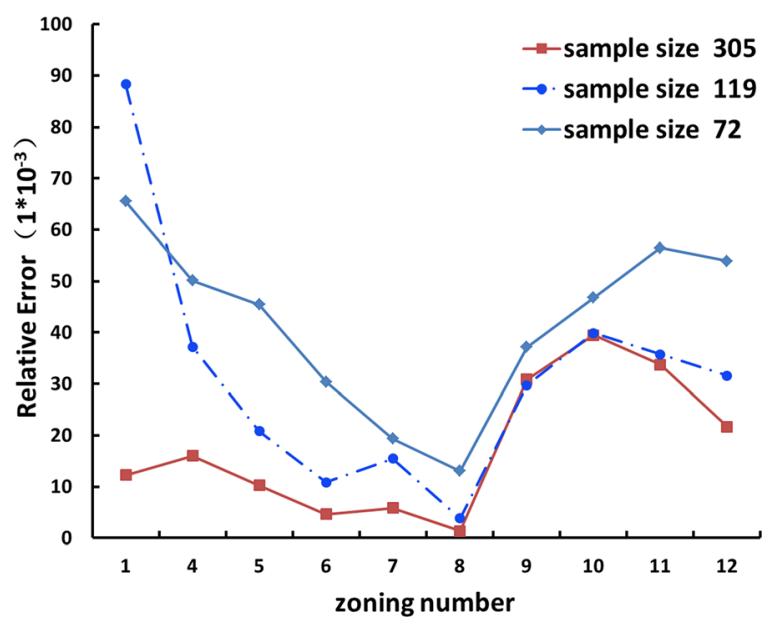

Fig. 6 Relative errors with different number of zones 
regulation of sample spatial distributions. For example, the errors of 119 and 142 are larger than the error of dataset of sample size 108. This is attributed to the high FDI reflected. The errors of global mean calculated by cell method and polygonal method are increased as the sample sizes decrease. Such as when the sample size is 72 , the errors are found to be the highest. It is seen that the self-organizing dual-zoning method is the most stable way to calculate the global mean; even the sample size is small, the errors are relatively accurate and stable.

The impact of number of zones on the dual-zoning method

In the preceding section, the self-organizing dual-zoning method was shown to be the optimal method for calculating the global mean. In this method, the number of zones is chosen based on Eq. (4). The number of zones to divide is an important factor influencing the efficiency. To further investigate the effect of the zoning number on the results, we performed an evaluation using three preferential sampling datasets.

When the number of zones is 1 , the mean calculation method is same as the arithmetic mean method. Thus, together with one zone, other numbers of zones ranging from 4 to 12 were selected based on time and working capacity constraints. In Fig. 6, it appeared that the error initially decreases with increasing zoning number, reaches a minimum value at eight zones, and increases thereafter. It is apparent that the number of zones has an influence on the mean calculation. When the number of zones is relatively less, the influence of the hybrid distance is small, leading to poor homogeneity within the zone. Thus, the zone efficiency is poor. In contrast, the results will be degraded to polygonal method when the number of zones increases to a certain extent. Because the number of zones determines the sample points in the zones, it affects the weights of the sampling sites when estimating the global mean.

To analyze how variations in number of zones lead to different mean errors, the layer with eight zones and the layer with ten zones for the 305 original sampling dataset were chosen to compare the highest and lowest error results. By overlaying the two layers, 33 intersection subzones were obtained. Each sampling site located in the subzone has two weights to estimating the global mean, one is calculated from the layer with eight zones, and the other is calculated from the layer with ten zones. Thus, for each subzone, there are also two weights corresponding to each layer, calculated by summing

Table 2 The mean and weight of each subzone

\begin{tabular}{|c|c|c|c|c|c|c|c|c|c|}
\hline Subzone & Mean & $\begin{array}{l}\text { Weight from } \\
\text { layer with ten } \\
\text { zones }\end{array}$ & $\begin{array}{l}\text { Weight from layer } \\
\text { with eight zones }\end{array}$ & $\begin{array}{l}\text { Weight } \\
\text { difference }\end{array}$ & Subzone & Mean & $\begin{array}{l}\text { Weight from } \\
\text { layer with ten } \\
\text { zones }\end{array}$ & $\begin{array}{l}\text { Weight from layer } \\
\text { with eight zones }\end{array}$ & $\begin{array}{l}\text { Weight } \\
\text { difference }\end{array}$ \\
\hline 1 & 547.88 & $8.85 \%$ & $4.08 \%$ & $-4.77 \%$ & 18 & 576.31 & $4.13 \%$ & $4.03 \%$ & $-0.10 \%$ \\
\hline 2 & 426.97 & $3.28 \%$ & $1.60 \%$ & $-1.68 \%$ & 19 & 717.08 & $0.34 \%$ & $0.24 \%$ & $-0.10 \%$ \\
\hline 3 & 590.93 & $3.12 \%$ & $1.73 \%$ & $-1.39 \%$ & 20 & 682.12 & $1.01 \%$ & $0.93 \%$ & $-0.08 \%$ \\
\hline 4 & 490.97 & $2.08 \%$ & $0.96 \%$ & $-1.12 \%$ & 21 & 568.39 & $0.52 \%$ & $0.44 \%$ & $-0.08 \%$ \\
\hline 5 & 514.43 & $3.28 \%$ & $2.40 \%$ & $-0.87 \%$ & 22 & 356.07 & $0.33 \%$ & $0.29 \%$ & $-0.04 \%$ \\
\hline 6 & 378.46 & $3.63 \%$ & $2.93 \%$ & $-0.70 \%$ & 23 & 601.04 & $0.13 \%$ & $0.13 \%$ & $0.00 \%$ \\
\hline 7 & 655.85 & $4.36 \%$ & $3.75 \%$ & $-0.61 \%$ & 24 & 482.89 & $0.32 \%$ & $0.37 \%$ & $0.05 \%$ \\
\hline 8 & 380.38 & $0.91 \%$ & $0.32 \%$ & $-0.59 \%$ & 25 & 488.61 & $0.64 \%$ & $0.73 \%$ & $0.09 \%$ \\
\hline 9 & 358.75 & $1.36 \%$ & $0.87 \%$ & $-0.49 \%$ & 26 & 448.53 & $0.93 \%$ & $1.12 \%$ & $0.19 \%$ \\
\hline 10 & 598.64 & $1.04 \%$ & $0.58 \%$ & $-0.46 \%$ & 27 & 510.08 & $2.56 \%$ & $2.93 \%$ & $0.36 \%$ \\
\hline 11 & 576.13 & $11.53 \%$ & $11.11 \%$ & $-0.43 \%$ & 28 & 437.31 & $0.27 \%$ & $0.73 \%$ & $0.46 \%$ \\
\hline 12 & 680.67 & $1.68 \%$ & $1.44 \%$ & $-0.23 \%$ & 29 & 529.35 & $1.20 \%$ & $2.16 \%$ & $0.96 \%$ \\
\hline 13 & 622.88 & $0.52 \%$ & $0.29 \%$ & $-0.23 \%$ & 30 & 264.31 & $4.69 \%$ & $6.09 \%$ & $1.40 \%$ \\
\hline 14 & 605.39 & $0.52 \%$ & $0.29 \%$ & $-0.23 \%$ & 31 & 493.35 & $6.31 \%$ & $8.78 \%$ & $2.47 \%$ \\
\hline 15 & 657.95 & $0.34 \%$ & $0.13 \%$ & $-0.21 \%$ & 32 & 495.14 & $9.83 \%$ & $13.18 \%$ & $3.35 \%$ \\
\hline 16 & 724.95 & $1.34 \%$ & $1.16 \%$ & $-0.19 \%$ & 33 & 514.72 & $6.25 \%$ & $11.65 \%$ & $5.40 \%$ \\
\hline 17 & 291.87 & $12.70 \%$ & $12.58 \%$ & $-0.12 \%$ & & & & & \\
\hline
\end{tabular}


corresponding weights of sampling points located in it. Also the difference between weight from layer with eight zones and that from layer with ten zones of each subzone can be gotten to represent the discrepancy of the different number of zones. The arithmetic mean, the two weights from different layer, and their difference of each subzone were calculated and shown in Table 2. Using a grayscale map for the simulation reference dataset as a base map, Fig. 7 shows the weight difference of each point using different colors and point sizes. The point size is proportional to the absolute value of the weight difference, and point colors ranging from green to red denote negative to positive differences, respectively.

The distribution of the weight differences is clearly identifiable. A majority of points with intense spatial aggregation have weight differences that are negative and have large absolute values, whereas almost all points that sparsely distributed have positive differences. So a certain relationship between the distribution of weight differences and the aggregation of points in geographic space exists, and it is clear that the spatial aggregation of points is one of the factors influencing the results for different number of zones.

In addition, the distribution in feature space also influences the weights. The Hot Spot Analysis tool of ArcGIS v. 9.3., which can identify spatial clusters using the Getis-Ord Gi* statistic, was used to evaluate the distribution in feature space of the sample with 305 points. The $Z$ score obtained is 7.36 , indicating a spatial clustering of high values in the feature space. The base map also demonstrates this effect. The weight differences in areas with high values are less than those in the low-value areas. Thus, it is determined that the accuracy of the weight assignments or the number zones is influenced by the space aggregation and value attributes in feature space. When performed for samples having a spatial clustering of high values, the zoning number should be chosen according to Eq. (4), which can guarantee the rationality of the zoning.

\section{Conclusions}

It was shown that the arithmetic mean method is not suited to the special properties of preferential sampling data and it is apt to result in a highly biased calculation. Clearly, this method can reliably produce an accurate mean estimate only for a specific sample size and reasonable sample layout. In addition, the cell and polygonal methods can decrease the errors of the mean calculation to a certain extent; however, these methods are influenced by feature deviations, and when the sample size is small, the results contain substantial errors. The most important conclusion of this paper is that regardless of the feature deviation and sample size, the global
Fig. 7 Weight difference of sampling points between eight zones and ten zones

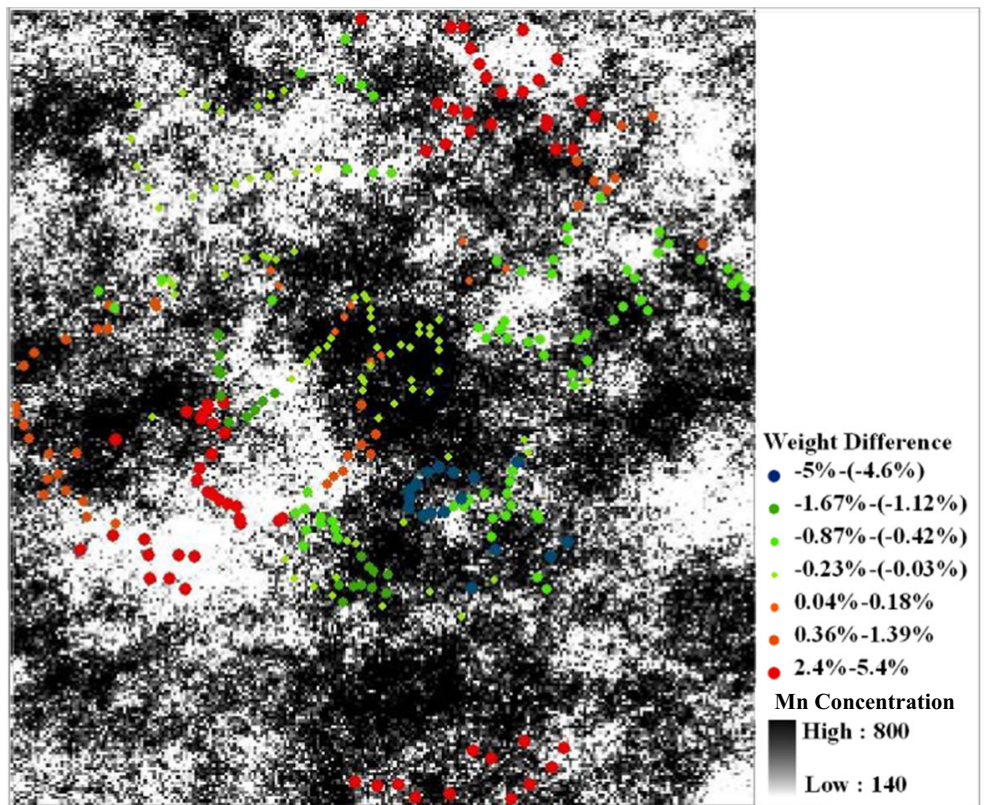


means estimated by the self-organizing dual-zoning method are more robust than those obtained using the other three methods. This difference is attributed to the fact that the self-organizing dual-zoning method fully takes into account the distribution of the geographic and feature space. However, for the case that some subareas with sharp different characteristics have no sampling points, this method cannot either get a good estimation for the global mean. So, the preferential sampling can be treated by self-organizing dual-zoning method should at least have one sampling points in subareas with sharp different characteristics. And also, in the mean calculation using this method, the number of zones should be properly set to efficiently address the heterogeneity of population.

Acknowledgments This study was funded by the National Natural Science Foundation of China (No. 40971237 and No. 41201173) and the Open Fund of National Engineering Research Center for Information Technology in Agriculture (No. KF2012N08-055).

\section{References}

Botta-Dukát, Z., Kovács-Láng, E., Rédei, T., Kertész, M., \& Garadnai, J. (2007). Statistical and biological consequences of preferential sampling in phytosociology: theoretical considerations and a case study. Folia Geobotanica, 42(2), 141152.

Deutsch, C. (1989). DECLUS: a FORTRAN 77 program for determining optimum spatial declustering weights. Computers \& Geosciences, 15(3), 325-332.

Diggle, P. J., Menezes, R., \& Su, T. L. (2010). Geostatistical inference under preferential sampling. Journal of the Royal Statistical Society: Series C (Applied Statistics), 59(2), 191232.

Dubois, G., \& Saisana, M. (2002). Optimizing spatial declustering weights - comparison of methods (pp. 479-484). BerlinGermany: In Proceedings of the Annual Conference of the International Association for Mathematical Geology.

Goovaerts, P. (1997). Geostatistics for natural resources evaluation (pp. 393-395). New York: Oxford University Press.

Gupta, S., \& Shabbir, J. (2007). On the use of transformed auxiliary variables in estimating population mean by using two auxiliary variables. Journal of Statistical Planning and Inference, 137(5), 1606-1611.

Isaaks, E. H., \& Srivastava, R. M., 1989. Applied geostatistics .Oxford University Press, 561pp.
Jiao, L., Liu, Y., \& Zou, B. (2011). Self-organizing dual clustering considering spatial analysis and hybrid distance measures. Science China Earth Sciences, 54(8), 1268-1278.

Journel, A. G. (1983). Nonparametric estimation of spatial distributions. Journal of the International Association for Mathematical Geology, 15(3), 445-468.

Kamiran, F., \& Calders, T., 2010. Classification with no discrimination by preferential sampling. In Proc. Benelearn.

Kohonen, T. (1990). The self-organizing map. Proceedings of the IEEE, 78(9), 1464-1480.

Li, L., Wang, J., Cao, Z., \& Zhong, E. (2008). An informationfusion method to identify pattern of spatial heterogeneity for improving the accuracy of estimation. Stochastic Environmental Research and Risk Assessment, 22(6), 689704.

Lin, C. R., Liu, K. H., \& Chen, M. S. (2005). Dual clustering: integrating data clustering over optimization and constraint domains. Knowledge and Data Engineering, IEEE Transactions on, 17(5), 628-637.

Menezes, R., 2009. Clustering and preferential sampling, two distinct issues in geostatistics. In XVII Annual Congress of the Portuguese Society of Statistics.

Merckx, B., Steyaert, M., Vanreusel, A., Vincx, M., \& Vanaverbeke, J. (2011). Null models reveal preferential sampling, spatial autocorrelation and overfitting in habitat suitability modelling. Ecological Modelling, 222(3), 588-597.

Michalcová, D., Lvončík, S., Chytrý, M., \& Hájek, O. (2011). Bias in vegetation databases? A comparison of stratified-random and preferential sampling. Journal of Vegetation Science, 22(2), 281-291.

Olea, R. A. (2007). Declustering of clustered preferential sampling for histogram and semivariogram inference. Mathematical Geology, 39(5), 453-467.

Rao, T. J. (1981). On a class of almost unbiased ratio estimators. Annals of the Institute of Statistical Mathematics, 33(1), 225231.

Shabbir, J., \& Yaab, M. Z. (2003). Improvement over transformed auxiliary variable in estimating the finite population mean. Biometrical Journal, 45(6), 723-729.

Tai, C. H., Dai, B. R., \& Chen, M. S. (2007). Incremental clustering in geography and optimization spaces. In Advances in Knowledge Discovery and Data Mining (pp. 272-283). Berlin Heidelberg: Springer.

Thiessen, A. H. (1911). Precipitation averages for large areas. Monthly Weather Review, 39(7), 1082-1089.

Wang, J. F., Christakos, G., \& Hu, M. G. (2009). Modeling spatial means of surfaces with stratified nonhomogeneity. Geoscience and Remote Sensing, IEEE Transactions on, 7(12), 4167-4174.

Wang, J., Haining, R., \& Cao, Z. (2010). Sample surveying to estimate the mean of a heterogeneous surface: reducing the error variance through zoning. International Journal of Geographical Information Science, 24(4), $523-543$. 